\author{
Abstracta Iranica \\ Abstracta Iranica Revue bibliographique pour le domaine irano-aryen \\ Volume 37-38-39 | 2018 \\ Comptes rendus des publications de 2014-2016
}

\title{
Julia Gonnella, Friederike Weis, Christoph Rauch (éd.). The Diez Album. Contexts and Contents
}

\section{Frantz Chaigne}

\section{(2) OpenEdition}

\section{Journals}

Édition électronique

URL : http://journals.openedition.org/abstractairanica/43313

DOI : 10.4000/abstractairanica.43313

ISBN : 1961-960X

ISSN : 1961-960X

Éditeur :

CNRS (UMR 7528 Mondes iraniens et indiens), Éditions de l'IFRI

Référence électronique

Frantz Chaigne, « Julia Gonnella, Friederike Weis, Christoph Rauch (éd.). The Diez Album. Contexts and Contents », Abstracta Iranica [En ligne], Volume 37-38-39 | 2018, document 27, mis en ligne le 30 décembre 2018, consulté le 02 octobre 2020. URL : http://journals.openedition.org/abstractairanica/ 43313 ; DOI : https://doi.org/10.4000/abstractairanica.43313

Ce document a été généré automatiquement le 2 octobre 2020.

Tous droits réservés 


\title{
Julia Gonnella, Friederike Weis, Christoph Rauch (éd.). The Diez Album. Contexts and Contents
}

\author{
Frantz Chaigne
}

\section{RÉFÉRENCE}

Julia Gonnella, Friederike Weis, Christoph Rauch (éd.). The Diez Album. Contexts and Contents. Leyde/Boston: Brill, 2016, 672 p., ISBN :978-90-04-32348-3

1 Le présent ouvrage regroupe les actes du colloque qui s'était tenu à Berlin du 2 au 5 juin 2013 autour des Albums Diez. Ces albums, conçus par un diplomate allemand en poste à Istanbul, sont composés de fragments de miniatures et de calligraphies de divers formats, coupés et collés. Les contributions regroupées dans le présent volume s'organisent en grandes parties cohérentes montrant la multiplicité des approches possibles pour ce monument qui autorise une pluralité de regards (historiens de l'art, codicologues, etc.). L'accent est certes porté sur les époques de production des miniatures, dessins et calligraphies; néanmoins, les contributions relatives à l'élaboration ultérieure de ces volumes et leur analyse trouvent toute leur place.

2 À une introduction signée par les trois éditeurs, succède une première partie intitulée "The Albums and Heinrich von Diez ». Cette partie historiographique est constituée de trois chapitres respectivement signés par Julian Raby, David Roxburgh et Christoph Rauch. La deuxième partie, "The Diez and the Topkapı Albums", permet de revenir avec des regards réactualisés sur une problématique délicate dans le domaine des arts du livre. Cette partie, composée de trois chapitres écrits par Lâle Uluç, Zeren Tanındı et Simon Rettig, met en exergue les apports des études iconographiques et calligraphiques. La troisième partie, «The Albums' Contents: From the Mongols to the Timurids ", contient des études de cas en iconographie, particulièrement éclairantes, rédigées par Charles Melville, Yuka Kadoi (cf. cr dans ce volume), Claus-Peter Haase, 
Barbara Brend et Filiz Çakır Philip. La quatrième partie, "The Albums' Contents: Drawings and Sketches", permet d'appréhender la dynamique de fabrication des images. Les articles la constituant sont dus aux plumes d'Yves Porter, Friederick Weis et Oliver Hahn. La pénultième partie, «Repatriations: The Diez Albums as a Source of Reconstrucive Lost Art " (articles de Robert Hillenbrand, Bernard O'Kane, Massumeh Farhad et Karin Rührdanz), permet de replacer ces pages dans un réseau de manuscrits prestigieux comme les grands Shahnameh mongol et jalayiride ou le Divan de Sultan Ahmad Jalayiride. La dernière partie, «Europe, China and Istanbul: The Albums in a Broader Perspective", élargit encore les perspectives via des articles de Gülru Necipoğlu, Chin-Ling Wang et Serpil Bağcı. Viennent ensuite les appendices dont des index détaillés. Il convient aussi d'insister sur la richesse de l'iconographie pour chaque article.

Ce volume mérite bien sûr d'être mis en rapport avec actes du colloque de 1980 organisé par Ernst Grube autour des albums de Topkapı (Islamic Art 1, 1981). On pourra ainsi à la fois comparer ces artefacts voisins, tout en appréhendant les évolutions de la recherche et de ses outils méthodologiques sur cette période de 35 ans.

\section{AUTEURS}

\section{FRANTZ CHAIGNE}

Chercheur associé, CNRS, Orient \& Méditerranée-«Islam médiéval» 